\title{
Impact Predictions of Face Gear Rim Thicknesses on Mesh Stiffness of Face Gear Drives
}

\author{
H.S. Chen, Z.M.Q. Li, X.S. Liu, H. Wu, J. Wang \\ College of Mechanical and Electrical Engineering \\ Nanjing University of Aeronautics and Astronautics \\ China
}

\begin{abstract}
Mesh stiffness is one of fundamental parameters of face gear dynamics. Thus, a calculation solution of mesh stiffness of face gear drives was constructed by finite element method. The impact of face gear rim thicknesses on mesh stiffness was predicted. The analytical results indicate that a face gear rim is thicker, average mesh stiffness is larger, but changes of average mesh stiffness would slow versus changes of face gear rim thickness. The contributions are benefit to improve the design of face gear drives associated with low vibrations.
\end{abstract}

Keywords- face gear drives; tooth contact analysis; finite element method; gear mesh stiffness

\section{INTRODUCTION}

Face gear drives are addressed by many scholars due to its insensitive manufacture and alignment errors versus bevel gear drives [1 5]. Face gear dynamics is a part of studies of face gear drives, and mesh stiffness is one of the fundamental parameters of face gear dynamics. Mesh stiffness calculation solutions of spur and bevel gear drives have been constructed [6 9]. However, mesh stiffness of face gear drives with a spur gear is yet to be further researched. Thus, a constructed solution of face gear drives mesh stiffness was provided, and the impact of face gear rim thicknesses on mesh stiffness is predicted in this study. It is based on the contact finite element method (FEM) for face gear drives. The geometric modelling of a face gear drive is constructed. The mesh stiffness solution and data processing were analysed in FEM software. The deformations of different rims are also computed. And then mesh stiffness of face gear drives of different rim thicknesses are obtained. The conclusions of this study indicate that a thicker face gear rim will lead to larger average mesh stiffness, but changes of average mesh stiffness would slow versus changes of face gear rim thickness. Therefore, the paper provides an approach for calculating mesh stiffness of face gear drives, and the impact prediction is meaningful for its design process.

\section{CONSTRUCTION OF A GEOMETRIC MODELLING SOLUTION}

\section{A. Equations of Face Gear Tooth Surface}

Face gears are manufactured by involute generating cutters. The coordinate system of a cutter [10] is given by Fig. 1. $\varphi s$ is the rotational angle of the cutter. The tooth surface vector equations [10] of the cutter are expressed as:

$$
\vec{r}_{S}\left(u_{S}, \theta_{S}\right)=\left[\begin{array}{llll}
x_{S} & y_{S} & z_{S} & t
\end{array}\right]^{T}=\left[\begin{array}{c} 
\pm r_{b S}\left[\sin \left(\theta_{S 0}+\theta_{S}\right)-\theta_{S} \cos \left(\theta_{S 0}+\theta_{S}\right)\right] \\
-r_{b S}\left[\cos \left(\theta_{S 0}+\theta_{S}\right)+\theta_{S} \cos \left(\theta_{S 0}+\theta_{S}\right)\right] \\
u_{S} \\
1
\end{array}\right]
$$

Where $r_{\mathrm{bs}}$ is the base circle radius of the cutter; $u_{\mathrm{S}}$ and $\theta_{\mathrm{S}}$ are the axial parameter and angle parameter of a point on cutter surface, respectively; $\varphi_{\mathrm{S}}$ is the rotational angle of the gear cutter. $\theta_{\mathrm{S} 0}$ is an angle parameter from the symmetric plane of the tooth space to the initial point of the involute; " \pm " corresponds to the involutes at the two sides of the tooth space, namely $\gamma-\gamma$ and $\beta-\beta$, respectively.

Unit normal of the cutter tooth surface [10] is expressed as:

$$
\vec{n}_{S}=\left[\begin{array}{lll}
n_{S x} & n_{S y} & n_{S z}
\end{array}\right]^{T}=\frac{\partial \vec{r}_{S} / \partial \theta_{S} \times \partial \vec{r}_{S} / \partial u_{S}}{\left|\partial \vec{r}_{S} / \partial \theta_{S} \times \partial \vec{r}_{S} / \partial u_{S}\right|}=\left[\begin{array}{c}
\mp \cos \left(\theta_{S 0}+\theta_{S}\right) \\
-\sin \left(\theta_{S 0}+\theta_{S}\right) \\
0
\end{array}\right]
$$

The processing coordinate systems of an orthogonal face gear are presented in Figure 2. There are four coordinate systems, which are two fixed ones for the cutter $\mathrm{S}$ and the face gear 2, named $O_{\mathrm{S} 0} x_{\mathrm{S} 0} y_{\mathrm{S} 0} z_{\mathrm{S} 0}$ and $O_{2} x_{20} y_{20} z_{20}$, respectively; two rotating ones for the cutter $\mathrm{S}$ and the face gear 2, named $O_{\mathrm{S}} x_{\mathrm{S}} y_{\mathrm{S}} z_{\mathrm{S}}$ and $\mathrm{O}_{2} x_{2} y_{2} z_{2}$, respectively. The motion relationship of the gear shaper and a face gear is shown in Figure 2 (a).

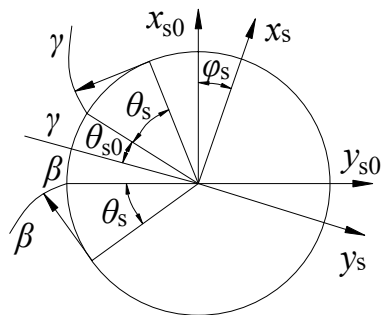

FIGURE I. THE COORDINATE SYSTEM OF THE CUTTER TOOTH SURFACE [10].

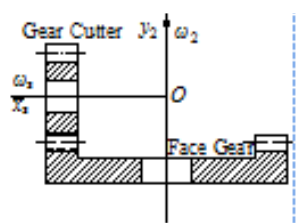

(a)

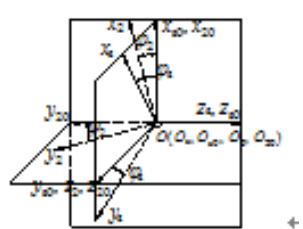

(b)
FIGURE II. THECOORDINATE SYSTEMOF FACE GEARS MACHINE [10] 
Tooth surface equations of a face gear are deduced by the conversion relationship from coordinate system $\mathrm{S}_{\mathrm{S}}$ to coordinate system $\mathrm{S}_{2}$. They are expressed [10] as:

$$
\left.\begin{array}{l}
\vec{r}_{2}\left(u_{S}, \theta_{S}, \varphi_{S}\right)=\left[M_{2, S}\right] \vec{r}_{S}\left(u_{S}, \theta_{S}\right) \\
f\left(u_{S}, \theta_{S}, \varphi_{S}\right)=0
\end{array}\right\}
$$

Where $\left[M_{2}, \mathrm{~s}\right]$ represents the transformation matrix of the two coordinate systems, and the second equation can be achieved by the correct meshing conditions. Therefore, the tooth surface equations can be expressed[10] as below:

$$
\vec{r}_{2}\left(u_{S}, \theta_{S}\right)=\left[\begin{array}{lll}
x_{2} & y_{2} & z_{2}
\end{array}\right]^{T}=\left[\begin{array}{c}
r_{b S}\left[\cos \varphi_{2}\left(\sin \varphi_{\theta} \mp \theta_{S} \cos \varphi_{\theta}\right)-\frac{\sin \varphi_{2}}{q_{2 S} \cos \varphi_{\theta}}\right] \\
-r_{b S}\left[\sin \varphi_{2}\left(\sin \varphi_{\theta} \mp \theta_{S} \cos \varphi_{\theta}\right)+\frac{\cos \varphi_{2}}{q_{2 S} \cos \varphi_{\theta}}\right.
\end{array}\right]
$$

Where $q_{2 \mathrm{~S}}$ is the gear ratio of the face gear and the gear cutter; $\varphi_{2}=q_{2 \mathrm{~S}} \varphi_{2} ; \varphi_{\theta}=\varphi_{\mathrm{S}} \pm\left(\theta_{\mathrm{S} 0}+\theta_{\mathrm{S}}\right)$.

\section{B. Tooth Contact Analysis}

The point contact face gear drives are studied because of unbalanced loading of line contact. A medium gear is a gear that can meet the line contact with the face gear and the pinion at same time. It is an imaginary gear. Its geometry parameters are always consistent with the cutter, and the tooth profile coordinate is shown in Figure 3 [11]. In Figure 3, $r_{\mathrm{b}}$ is the base circle radius of the medium gear; $r_{\mathrm{k}}$ is the radius vector of a point on the tooth profile, named $k . \theta_{\mathrm{k}}$ is the angle between $r_{\mathrm{k}}$ and $y$.

Hence, the tooth profiles equations [11] of the medium gear are expressed as:

$$
\left\{\begin{array}{l}
x_{m}= \pm r_{k} \sin \theta_{k} \\
y_{m}=r_{k} \cos \theta_{k} \\
z_{m}=u_{k}
\end{array}\right.
$$

Where $u_{\mathrm{k}}$ is a variable of the tooth profile equations of the medium gear in the tooth width direction. "+" represents the left tooth profile; "-" represents the right tooth profile in $x_{\mathrm{m}}$. Hence the homogeneous equation of the tooth profile is

$$
\boldsymbol{R}_{\boldsymbol{m}}=\left[\begin{array}{llll}
x_{m}, & y_{m}, z_{m}, 1
\end{array}\right]^{T} .
$$

The coordinate systems of the point contact face gear drives are shown in Figure4 [11]. In the coordinate systems, $O_{\mathrm{g}} X_{\mathrm{g}} Y_{\mathrm{g}} Z_{\mathrm{g}}$ and $O_{\mathrm{g}}{ }^{\prime} X_{\mathrm{g}}^{\prime} Y_{\mathrm{g}}^{\prime} Z_{\mathrm{g}}^{\prime}$ are the static coordinate and mobile coordinate of the pinion, respectively; $O_{\mathrm{m}} X_{\mathrm{m}} Y_{\mathrm{m}} Z_{\mathrm{m}}$ and $O_{\mathrm{m}}{ }^{\prime} X_{\mathrm{m}}{ }^{\prime} Y_{\mathrm{m}} Z_{\mathrm{m}}$ ' are the static coordinate and mobile coordinate of the medium gear, respectively; $O_{\mathrm{f}} X_{\mathrm{f}} Y_{\mathrm{f}} Z_{\mathrm{f}}$ and $O_{\mathrm{f}}^{\prime} X_{\mathrm{f}}^{\prime} Y_{\mathrm{f}}^{\prime} Z_{\mathrm{f}}^{\prime}$ are the static coordinate and mobile coordinate of the face gear, respectively. The plane $O_{\mathrm{g}} X_{\mathrm{g}} Y_{\mathrm{g}}$ and $O_{\mathrm{m}} X_{\mathrm{m}} Y_{\mathrm{m}}$ are in the same plane. The distance between $O_{\mathrm{g}}$ and $O_{\mathrm{m}}$ is $a$. The distance between the plane $O_{\mathrm{m}} X_{\mathrm{m}} Z_{\mathrm{m}}$ and $O_{\mathrm{f}} X_{\mathrm{f}} Y_{\mathrm{f}}$ is ra, and it is equal to the radius of the addendum circle of the medium gear. The plane $O_{\mathrm{m}} X_{\mathrm{m}} Y_{\mathrm{m}}$ and $O_{\mathrm{f}} X_{\mathrm{f}} Z_{\mathrm{f}}$ are parallel, and the distance between them is $d$.

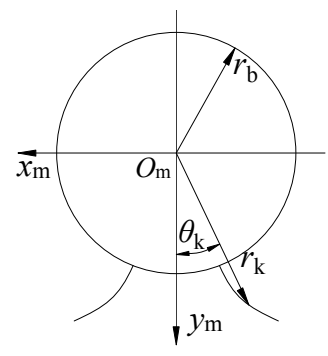

FIGURE III. TOOTH PROFILES COORDINATE OF THE MEDIUM GEAR.

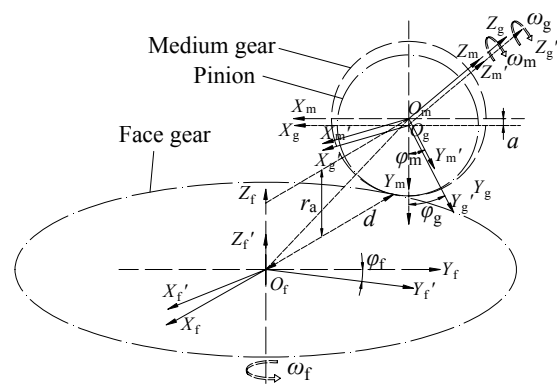

FIGURE IV. THE DRIVE COORDINATE SYSTEM OF POINT CONTACT FACE GEARS [11].

The surfaces equations and the envelope condition can be expressed[11] as eqn. (6) and eqn. (7). These equations are under the envelope principle.

$$
\begin{gathered}
\boldsymbol{R}_{m z}=\boldsymbol{M}_{f^{\prime} m} \boldsymbol{R}_{m} \\
\frac{\partial \boldsymbol{R}_{m z}}{\partial \alpha_{k}} \times \frac{\partial \boldsymbol{R}_{m z}}{\partial u_{k}} \cdot \frac{\partial \boldsymbol{R}_{m z}}{\partial \varphi_{m}}=0
\end{gathered}
$$

Where $\boldsymbol{M}_{\mathrm{f} \mathrm{m}^{\prime}}$ is the homogeneous matrix that the medium gear generates the face gear with envelope method. The surfaces equations of the medium gear can be expressed as eqn (8)The surfaces are enveloped by the spur gear. The envelope condition is expressed as eqn(9) [11].

$$
\begin{gathered}
\boldsymbol{R}_{g z}=\boldsymbol{M}_{m^{\prime} g^{\prime}} \boldsymbol{R}_{g} \\
\frac{\partial \boldsymbol{R}_{g z}}{\partial \alpha_{k}} \times \frac{\partial \boldsymbol{R}_{g}}{\partial u_{k}} \cdot \frac{\partial \boldsymbol{R}_{g z}}{\partial \varphi_{m}}=0
\end{gathered}
$$

Where $\boldsymbol{R}_{\mathrm{g}}=\boldsymbol{R}_{\mathrm{m}}, \boldsymbol{M}_{\mathrm{m} \mathrm{g}^{\prime}}$ is the homogeneous matrix when the pinion envelopes the medium gear. The four equations eqn(6), (7), (8) and (9) are contact equations of a face gear drive.

\section{Geometry Model of a Face Gear}

Discrete data points of the tooth surface can be solved from the equations of the face gear tooth surface by numerical calculation. The parameters of the face gear drive are shown in Table 1. The gear tooth surface can therefore be simulated as presented in Figure5. In the picture, the top land is sharpened at the inner radius and the dedendum is undercut at the outside radius. The contact points can be resolved as shown in Figure5 according to the above contact equations. To optimize the transmission, an appropriate face gear width like in Figure6 is chosen so that the contact points are on the middle of face width. Then a three-dimensional solid model can be simulated with the face gear surface in Figure6in software. The model of 
the face gear is shown in Figure7. Here the length of its rim is set as $0.5 \mathrm{~mm}$ to approximate the situation of no rim.

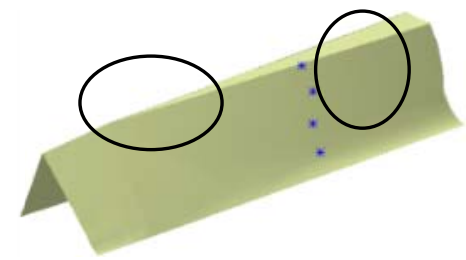

FIGURE V. TOOTH SURFACE SIMULATION.

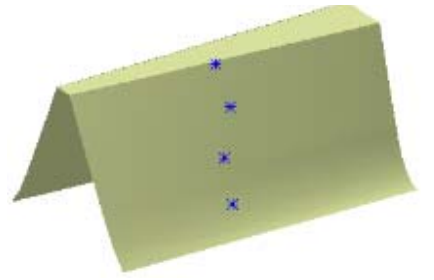

FIGURE VI. TOOTH CONTACT ANALYSIS.

TABLE I. THE FACE GEAR DRIVE PARAMETERS.

\begin{tabular}{|l|c|c|}
\hline \multirow{2}{*}{ Description } & \multicolumn{2}{|c|}{ Values } \\
\cline { 2 - 3 } & Spur gear & Face gear \\
\hline Module (mm) & 4 & 4 \\
\hline Number of teeth & 24 & 96 \\
\hline Pressure angle ( $\left.{ }^{\circ}\right)$ & 20 & 20 \\
\hline Tooth width (mm) & 30 & 30 \\
\hline Modification coefficient & 0 & 0 \\
\hline
\end{tabular}

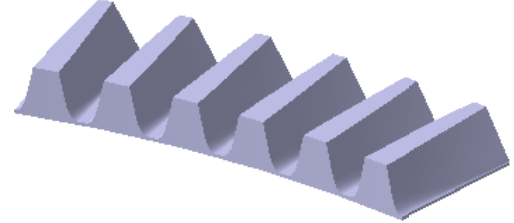

FIGURE VII. THE FACE GEAR SOLID MODEL

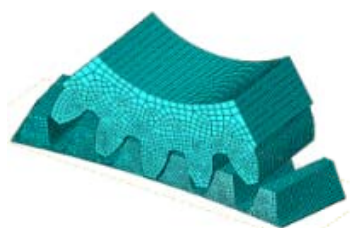

FIGURE VIII.

FEM MODEL CONSTRAINT

\section{THE LTCA OF A FACE GEAR DRIVE WITHOUT A RIM}

\section{A. The FEM Model}

Reduced integration element C3D8R is applied to the FEM model of the face gear drive. A partial face gear drive model is chosen to reduce the amount of computations and take face gear rim thickness into consideration. The FEM model is shown in Figure8.

Rigid coupling is created between the references points on the gear axis and the faces, as well as the inner ring and the pinion profile. This is because three-dimension solid elements do not have rotational degree of freedom in the FEA software. Afterwards, rotational velocity and torque can be applied on the reference points. The loads will be transferred to the FE models of spur gear and face gear. Thereby, the motion of the face gear driven by the spur gear can be simulated.

\section{B. Preprocessors of the FEM Analysis Model}

The preprocessors of a face gear driveFEM model include how to select the grid element, the settings of the analysis type, loads, boundary conditions, etc. All of these determine whether the results are accurate. Therefore, they are the key technologies. The main steps are:

(1) Definition of material: The elastic modulus and Poisson ratio of the two gears material are all set as $206 \mathrm{GPa}$ and 0.3 , respectively.

(2) Analysis Type: Analytical process is divided into two steps to ensure convergence of tooth contact analysis; they are two general analysis steps.

(3) Definition of contact: Define the contact tooth surfaces as contact pairs, and set the friction coefficient as 0.1 in contact property options.

(4) Boundary conditions and loads: In the first analysis step, the face gear is completely fixed and the spur gear is fixed with the exception of the rotational degree of freedom of its rotational axis. At the same time, apply a little displacement on the spur gear around the axis to get the perfect contact between two gears and accelerate the convergence; In the second analysis step, the constraints of the face gear are the same as the last step. At the same time, release the rotational freedom of degree of the spur gear and apply a $200 \mathrm{~N} \cdot \mathrm{m}$ torque on the reference point of the spur gear. The load increases to the maximum linearly in the mode of analysis step time.

\section{Results of the Finite Element Analysis}

In the paper, the meshing process of a single gear pair is divided into fifteen positions. The face gear rotates 0.5 degrees at every position. The loads and deformation of these positions can be calculated by FEM software.

The middle tooth surface of the gear sectors is analyzed, and the tooth contact force distribution is shown in Figure9. The picture shows that the contact area of the face gear is a long ellipse and it leans on the tooth surface when a load is applied. The total elastic deflection of the gear tooth is exported as a field variable and presented in Figure 10. The time varying contact forces can be picked up when the total contact forces of middle gear surface are regarded as history variable. The image is given in Figure 11. The ultimate iterative result of the contact force and the maximum deflection of the tooth surface are labelled as the force and the deformation, respectively. They are shown in Table 2.

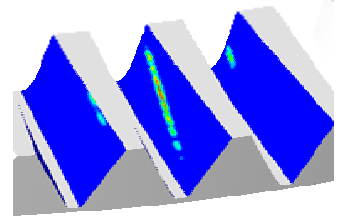

FIGURE IX. CONTACT FORCE DISTRIBUTION. 


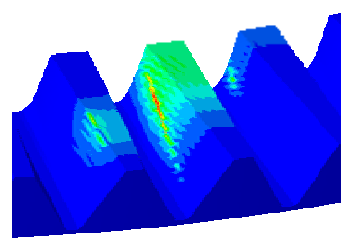

FIGURE X. TOOTH SURFACE DEFLECTION DISTRIBUTION.

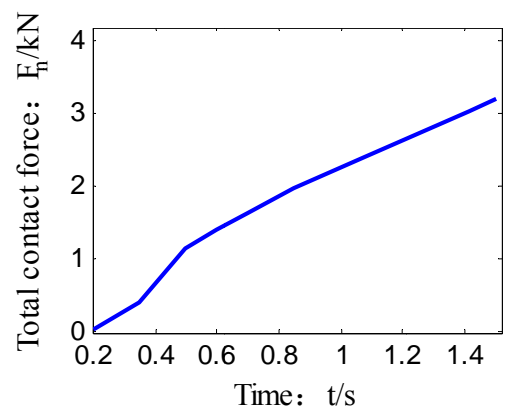

FIGURE XI. CONTACT FORCE CURVE.

TABLE II. CONTACT FORCE AND DEFORMATION AT EVERY ANGLE POSITION.

\begin{tabular}{|c|c|c|c|c|c|}
\hline $\begin{array}{c}\text { Rotatio } \\
\text { nal } \\
\text { angle } \\
\mathbf{(}^{\circ}\end{array}$ & $\begin{array}{c}\text { Contac } \\
\mathbf{t} \\
\text { forces( } \\
\mathbf{N})\end{array}$ & $\begin{array}{c}\text { Deformati } \\
\text { on (mm) }\end{array}$ & $\begin{array}{c}\text { Rotatio } \\
\text { nal } \\
\text { angle } \\
\mathbf{(})\end{array}$ & $\begin{array}{c}\text { Contac } \\
\mathbf{t} \\
\text { forces( } \\
\mathbf{N})\end{array}$ & $\begin{array}{c}\text { Deformati } \\
\text { on (mm) }\end{array}$ \\
\hline $\mathbf{0}$ & 947.437 & $5.32 \times 10^{-3}$ & 4 & 3304.62 & $5.69 \times 10^{-3}$ \\
\hline $\mathbf{0 . 5}$ & 1311.23 & $4.62 \times 10^{-3}$ & 4.5 & 2926.75 & $5.65 \times 10^{-3}$ \\
\hline $\mathbf{1}$ & 1750.94 & $5.35 \times 10^{-3}$ & 5 & 2730.44 & $5.68 \times 10^{-3}$ \\
\hline $\mathbf{1 . 5}$ & 2476.49 & $5.92 \times 10^{-3}$ & 5.5 & 2071.68 & $4.88 \times 10^{-3}$ \\
\hline $\mathbf{2}$ & 2787.14 & $5.40 \times 10^{-3}$ & 6 & 1667.10 & $4.81 \times 10^{-3}$ \\
\hline $\mathbf{2 . 5}$ & 3170.46 & $5.52 \times 10^{-3}$ & 6.5 & 1352.99 & $5.15 \times 10^{-3}$ \\
\hline $\mathbf{3}$ & 3343.93 & $5.66 \times 10^{-3}$ & 7 & 1023.60 & $6.04 \times 10^{-3}$ \\
\hline $\mathbf{3 . 5}$ & 3395.48 & $5.77 \times 10^{-3}$ & & & \\
\hline
\end{tabular}

\section{THE INFLUENCE OF RIM THICKNESS ON FACE GEAR MESH STIFFNESS}

The contact ratio of the face gear drive is solved as 1.791 by referring to literature [10]. The parameters are shown in Table 1.

Face gear rims are taken to be $2.5 \mathrm{~mm}, 5 \mathrm{~mm}$ and $7.5 \mathrm{~mm}$, which are illustrated in Figure 12. The rims are separated from face gears to compute their deformation alone. Therefore, the total deformation of one face gear is divided into two parts, namely the deformation of gear teeth and its rim. In this step, the inner rings of these rims are fixed, and the torque is applied on the outer rings. The results are presented in Table 3.

Then, the total deformation of the three face gears can be added. The multi-tooth mesh stiffness curve can therefore be derived by overlapping the results of single mesh stiffness based on the contact ratio. The time-varying mesh stiffness curves and average mesh stiffness of three face gear drives are plotted in Figure 13 and Figure 14, respectively. The pictures show that a larger rim thickness will lead to larger face gear mesh stiffness, but changes of average mesh stiffness would slow versus changes of face gear rim thickness.
TABLE III. THE DEFORMATION OF THE RIMS.

\begin{tabular}{|c|c|c|c|}
\hline Rim thickness/mm & 2.5 & 5 & 7.5 \\
\hline Deformation/mm & $1.74 \times 10^{-2}$ & $8.59 \times 10^{-3}$ & $5.70 \times 10^{-3}$ \\
\hline
\end{tabular}

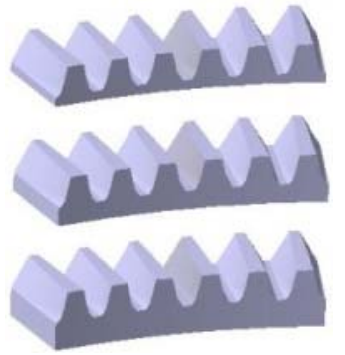

FIGURE XII.

THE FACE GEAR MODELS OF DIFFERENT RIMS.

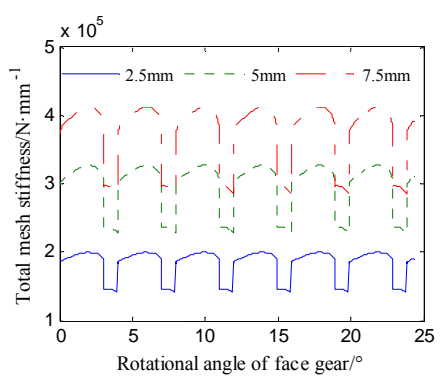

FIGURE XIII.

GEAR MESH STIFFNESS AT DIFFERENT RIM THICKNESSES

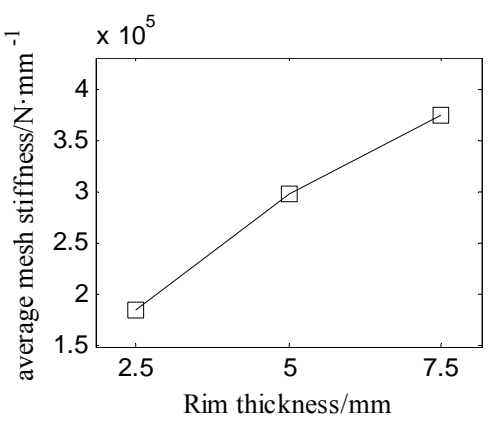

FIGURE XIV.

AVERAGE MESH STIFFNESS AT DIFFERENT RIM THICKNESSES

\section{CONCLUSION}

Three-dimensional solid model of a face gear was created with face gear tooth surface equations. The equations are derived by generating method. The FE model of a face gear drive was constructed by the element of 8-node hexahedral linear brick reduced integration element. The tooth surface contact force and total deflection was computed by LTCA analysis. The influence of different rims on face gear mesh stiffness was analyzed, and the results show that a face gear rim is thicker, average mesh stiffness is larger, but changes of average mesh stiffness would slow versus changes of face gear rim thickness. The solution constructed in this study is a feasible and effective method for gear mesh stiffness calculation of face gear drives. 


\section{ACKNOWLEDGMENTS}

The authors are grateful for the financial support provided by the National Natural Science Foundation of China under no.51105194 and no.51375226, and the Fundamental Research Funds for the Central Universities under no. NS2015049.

In addition, the authors declare that there is no conflict of interests regarding the publication of this article.

\section{REFERENCES}

[1] Zachary S. Henry. Bell helicopter advanced rotorcraft transmission (ART) program. Bell Helicopter Textron, Inc., Texas. NASA, CR 195497, 1995.6.

[2] Litvin F. L., Zhang Y., Wang J. C., et al. Design and geometry of facegear drives. Transactions of the ASME, Journal of Mechanical Design, 114(4), pp. 642-647,1992.

[3] Litvin F. L., Wang J. C., Bossler R. B. Application of face gear drives in helicopter transmissions. Journal of Mechanical Design, Transactions of the ASME, 116(3), pp. 672-676, 1994.

[4] M Guingand, JP De Vaujany, CY Jacquin. Quasi-static analysis of a face gear under torque, Comput. Methods Appl. Mech. Engrg, 194, pp. 43014318.2005 .

[5] Guanghu JIN, Rupeng ZHU, Zibing ZHU, et al. Analysis of the coupling vibration of a face gear transmission system, Mechanical Science and Technology for Aerospace Engineering, 28(1), pp. 124-128, 2009.

[6] Fakher C., Tahar F., Mohamed H. Analytical modelling of spur gear tooth crack and influence on gearmesh stiffness, European Journal of Mechanics A/Solids,28, pp. 461-468,2009.

[7] Zhaoyao SHI, Yan KANG, Jiachun LIN. Comprehensive dynamics model and dynamic response analysis of a spur gear pair based on gear pair integrated error, Journal of Mechanical Engineering, 46(17), pp. 5561,2010 .

[8] Yanzhong WANG, Shu WANG, Zhimin TIAN, et al. Research on loading tooth contact analysis of face gear transmission, Journal of Mechanical Transmission, 36(10), pp.4-7,2012.

[9] Xiaozhen LI, Rupeng ZHU, Zhengminqing LI, et al. Influence of Setting Error of Tool on Tooth Profile and Contact Point of Face Gear Drive. Transactions of Nanjing University of Aeronautics \&Astronautics.31(4), pp. 370 376, 2014.

[10] Rupeng ZHU. Reserch of meshing characteristic of face gear drives, Nanjing University of Aeronautics and Astronautics, Nanjing, 2000.

[11] Li Zhengminqing, Zhu Rupeng. Load Tooth Contact Analysis on Face Gear Driver, Journal of Nanjing University of Aeronautics \& Astronautics, 42(2), pp.219-223, 2010. 